\title{
Pseudogout, an unconventional imitator of the lumbosacral spine with associated chronic epidural hematoma: illustrative cases
}

\author{
Stephen Jaffee, MD, Seung Won Jeong, MD, Rocco Dabecco, DO, Shahed Elhamdani, MD, and Alexander Yu, MD \\ Department of Neurosurgery, Allegheny General Hospital, Pittsburgh, Pennsylvania
}

BACKGROUND Pseudogout, or calcium pyrophosphate dihydrate (CPPD) disease, is an inflammatory joint disease that most commonly involves the joints of the knees, ankles, and wrists. Pseudogout has also been known to involve the spine, especially the atlanto-occipital joint of the cervical spine, but there is limited documentation of its involvement in the lumbar spine. Though the atypical presentation of spinal pseudogout with findings consistent with discitis and epidural abscess has been documented, its presentation with associated chronic spinal epidural hematoma is a rare entity.

OBSERVATIONS The authors present two separate cases of pseudogout involvement of the lumbar spine, one case presenting with a clinical and radiographic picture consistent with discitis and epidural abscess and the other with radiographic and operative findings consistent with a chronic epidural hematoma.

LESSONS This case series demonstrates rare and atypical presentations of pseudogout within the lumbosacral spine.

https://thejns.org/doi/abs/10.3171/CASE21571

KEYWORDS pseudogout; spinal pseudogout; spinal epidural hematoma; spinal abscess; discitis

Pseudogout, also known as calcium pyrophosphate dihydrate (CPPD) disease, is a pathology that entails the accumulation of calcium pyrophosphate crystals in articular or periarticular spaces resulting in inflammatory joint disease. CPPD disease affects $4 \%-7 \%$ of adults in the United States, most commonly in patients $>60$ years of age. Risk factors for pseudogout include elderly age, prior spine surgery, spinal trauma, hemochromatosis, hypomagnesemia, hyperparathyroidism, hypercalcemia, hypothyroidism, acromegaly, ochronosis, and Wilson's disease. Pseudogout is classically diagnosed via polarized light microscopy of the synovial fluid of the affected joint demonstrating positively birefringent crystals with a characteristic rhomboid shape. ${ }^{1}$

Although pseudogout commonly causes acute or chronic inflammation in joints such as the knee, ankle, and hand, there has been limited documentation of pseudogout resulting in spinal pathology. Spinal CPPD disease most commonly involves the cervical spine, affecting the atlanto-occipital joint and resulting in cervico-occipital pain with fever, neck stiffness, and elevated inflammatory markers. Crystal deposition has also been documented in the lumbar spine and rarely the thoracic spine with a wide range of pathological presentations ranging from compressive radiculopathy to cauda equina syndrome. ${ }^{1}$ There also have been a few cases of spinal pseudogout mimicking infection/epidural abscess reported in the literature. However, there is limited literature on spinal epidural hematoma secondary to CPPD deposition.

We report two cases of spinal pseudogout presenting as epidural abscess and chronic lumbar epidural hematoma. We hope that additional case reports may aid in the understanding of the pathological profile of pseudogout of the spine and guide future clinicians in developing effective and efficient means of diagnosing and subsequently treating patients with this condition.

\section{Illustrative Cases}

\section{Case 1}

A 75-year-old female presented to the emergency department with progressive left lower extremity radiculopathy and paresthesias for the past 9 months. The patient had a previous surgical history of L4-5 laminectomy for neurogenic claudication secondary to stenosis.

ABBREVIATIONS CPPD = calcium pyrophosphate dihydrate; $C T$ = computed tomography; $E S R$ = erythrocyte sedimentation rate; MRI = magnetic resonance imaging INCLUDE WHEN CITING Published February 21, 2022; DOI: 10.3171/CASE21571.

SUBMITTED October 4, 2021. ACCEPTED October 20, 2021.

(C) 2022 The authors, CC BY-NC-ND 4.0 (http://creativecommons.org/licenses/by-nc-nd/4.0/). 

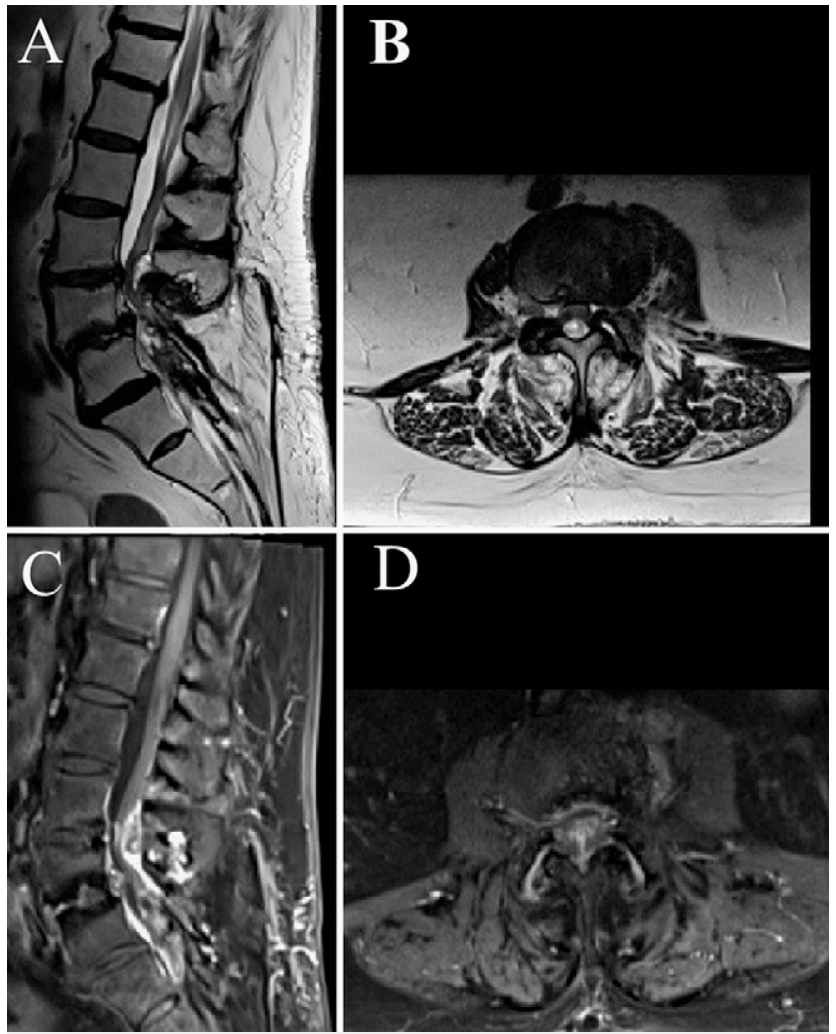

FIG. 1. A and B: Preoperative T2 sagittal and axial lumbar MRI scans, respectively. $\mathbf{C}$ and $\mathbf{D}$ : Preoperative $\mathrm{T} 1$ with contrast-enhanced sagittal and axial lumbar MRI scans, respectively. The scans show severe spinal canal stenosis at L3-4 secondary to a focally enhancing dorsal epidural collection as well as edema and enhancement of the intervertebral disc at L4-5, concerning for epidural abscess and discitis.

On examination, the patient had no weakness but had subjectively decreased sensation to light touch in her left lower extremity. Her blood work was significant for an elevated erythrocyte sedimentation rate (ESR) of 62. Magnetic resonance imaging (MRI) of the lumbar spine revealed severe spinal canal stenosis at L3-4 secondary to a focally enhancing dorsal epidural collection, as well as severe left foraminal stenosis at this level, edema and enhancement of the intervertebral disc at $L 4-5$, and clumping of nerve roots of the cauda equina and enhancement of the region, concerning for arachnoiditis. The patient underwent computed tomography (CT)-guided L4-5 disc aspiration due to concern for a possible infection, but no drainable fluid was found. Due to her progressive left lower extremity symptoms, elevated ESR, and area of enhancement on MRI concerning for an epidural abscess, the patient underwent an L3-4 laminectomy and foraminotomy (Fig. 1).

Intraoperatively, an unusual yellow-colored material within the epidural space and surrounding ligament was identified, which was concerning for a possible phlegmon. It was subsequently resected and sent for pathology. The procedure continued without any complications. The patient tolerated the procedure well and postoperatively had improvement of her left lower extremity radiculopathy and paresthesia. All samples from the operating room, including blood cultures, aspirated disc fluid, and the gross specimen, had negative results of culture analysis. Subsequent sequencing testing for
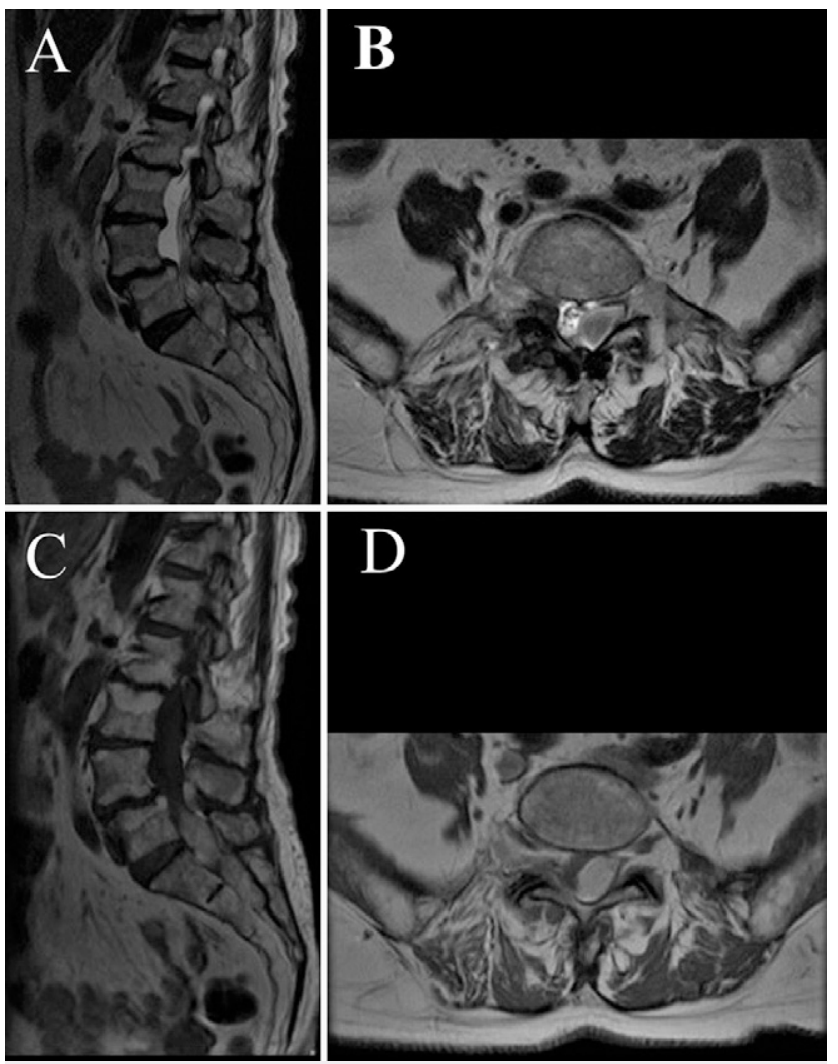

FIG. 2. A and B: Preoperative T2 sagittal and axial lumbar MRI scans, respectively. C and D: Preoperative T1 sagittal and axial MRI scans, respectively, without contrast enhancement. The scans show a left paracentral dorsal epidural lesion extending from $L 5$ to $S 2$ resulting in severe compression of the cauda equina.

definitive identification of a bacterial agent was also negative. Due to lack of a diagnosis, a polarized light study was completed, which demonstrated polarizable crystals most consistent with calcium pyrophosphate, and a diagnosis of pseudogout was made. The patient was started on colchicine therapy and had further improvement in her radicular symptoms.

\section{Case 2}

An 84-year-old female presented to the outpatient clinic with a 6-week history of lower back pain with bilateral lower extremity radiculopathy. The patient endorsed difficulty ambulating, standing, and rising from a seated position. Her past medical history was remarkable for breast cancer and skin cancer, in remission at the time, without any prior history of back pain. She sought surgical consultation after conservative management had failed, including oral steroids, physical therapy, and epidural steroid injections. Her physical examination was unremarkable except for an unsteady gait, and $\mathrm{MRI}$ of the lumbar spine revealed an epidural lesion extending from L5 to S2 (Fig. 2).

The patient was taken to the operating room for an S1-2 laminectomy and excision of the unknown mass. During the procedure, an extradural encapsulated mass was visualized extending from the L5 nerve root down to the S2 nerve root. Upon dissection of this mass from the dura, a dark motor oil-like fluid with a fibrous 
capsule was observed and subsequently drained, excised, and sent for pathological analysis. The patient did well postoperatively with resolution of her low back pain and bilateral lower extremity radiculopathy. Pathology described the gross specimen as tan-red and pink fibrous soft tissue and determined it to be an organized hematoma with dense fibrous tissue surrounding the collection of coagulated blood products. A polarized light study was also completed, and calcium pyrophosphate crystals were visualized within the dense fibrous tissue surrounding the hematoma, correlating with a diagnosis of pseudogout of the spine.

\section{Discussion}

Pseudogout can deposit in the intervertebral disc, joint capsule, facet cysts, synovial cysts, and spaces within the neural foramen, causing compressive pathological presentations. ${ }^{2-7}$ It has often been confused radiographically with calcified herniated disc, osteomyelitis/discitis with epidural phlegmon, or an extradural tumor., Patients can also present with elevated inflammatory markers, further complicating the initial presentation differential diagnosis and perhaps placing bias on an infectious etiology, a much more common diagnosis. ${ }^{8}$

MRI- and CT-guided biopsy plays a crucial role in diagnosing many spine pathologies that could cause compressive symptoms (i.e., infection, osteophyte formation, disc herniation, and tumor). Consideration of pseudogout as an alternative diagnosis for epidural abscess/collection in the setting of repeated sterile cultures and elevated inflammatory markers is imperative. Efforts should be made to collect samples for analysis under polarized light under these circumstances because this analysis may provide the necessary information to definitively diagnose the condition.

Dau et al. have demonstrated the low specificity of imaging studies and delayed diagnosis of spinal CPPD disease. ${ }^{9}$ Only $6 \%$ of their reported cases demonstrating radiographic findings consistent with CPPD disease; all others had negative imaging findings but eventually were diagnosed with the condition. Many patients had nonclassical presentations, with only $34 \%$ having peripheral chondrocalcinosis. This point highlights the need for specimen collection for definitive diagnosis, and specimen collection should be more commonplace as this pathology becomes more prevalent and better understood throughout the literature. ${ }^{9}$ To that point, recent literature suggests that CPPD disease of the spine is not limited to the extramedullary space, bone, and surrounding tissues. Gewolb et al. ${ }^{10}$ and Chakravorty et al. ${ }^{11}$ demonstrated an intramedullary lesion positive for CPPD disease, an extremely rare presentation, which further adds to the atypical presentations of this disease.

\section{Observations}

Although our first case demonstrated a documented representation and course of management of this pathology, our second case may be one of the few examples of CPPD disease correlating with chronic epidural hematoma. Spinal epidural hematomas are rare, with the annual incidence estimated at 0.1 per 100,000 people and $2 \%$ of all lesions of the spine. ${ }^{12}$ The chronic form is even more rare, representing $0.1 \%$ of spinal lesions. Epidural bleeding, however, is the most common type of bleeding occurring in the spinal column when compared with hematomas in the subdural and intramedullary spaces. Spinal epidural hematomas can be divided into three categories: secondary, spontaneous, and idiopathic. Secondary causes are usually related to spine surgery or other trauma, including hematomas developing from spinal injections. They can also be related to use of anticoagulants and coagulopathies, acquired and congenital, and associated with diseases, including Kummell's disease. Spontaneous epidural hematomas are rare, occurring in $0.3-0.9 \%$ of all space-occupying lesions in the epidural space. ${ }^{13}$ The term "idiopathic" is used when a causal relationship with an event or condition cannot be identified. Many theorize that these spontaneous hematomas may be caused by occult vascular malformations. The causal relationship between CPPD disease and chronic epidural hematoma is unknown, but it is postulated that tissue inflammation and neovascularization with concomitant vessel erosion may lead to formation of an epidural hematoma.

Many descriptions of the lesions involve either radiographic evidence of calcification or gross pathology description of yellow turbid fluid or chalky white appearance, but these findings are not specific for CPPD disease. ${ }^{1-11}$ As evidenced by the various presentations throughout the literature, there is no single pathognomonic finding except for visualization of positively birefringent rhomboid crystals on polarized microscopy. Therefore, polarized light analysis should become more commonplace in spinal pathology.

\section{Lessons}

The variable presentations of this condition reflect the invasive and locally destructive capacity of CPPD crystal deposition. More research needs to be completed to fully understand the mechanism that causes the accumulation and deposition of CPPD crystals in the spine, because it is substantially less common than in other joints (i.e., the elbow, knee, and hand). As physicians become more aware of this pathology, the time to diagnosis could be improved with more cost-effective management strategies and treatment options to avoid subjecting patients to unnecessary surgeries, antibiotic treatments, or readmissions. As it stands, immunomodulatory and anti-inflammatory medications have been the gold standard of treatment for CPPD disease. Further work must be done in conjunction with our rheumatology, pathology, and radiology colleagues to create more standardized diagnostic criteria and treatments for patients presenting with this condition.

\section{References}

1. Ivory $D$, Velázquez $C R$. The forgotten crystal arthritis: calcium pyrophosphate deposition. Mo Med. 2012;109(1):64-68.

2. Lam HY, Cheung KY, Law SW, Fung KY. Crystal arthropathy of the lumbar spine: a report of 4 cases. J Orthop Surg (Hong Kong). 2007;15(1):94-101.

3. Srinivasan V, Kesler H, Johnson M, Dorfman H, Walter K. Tophaceous pseudogout of the thoracic spine. Acta Neurochir (Wien). 2012;154(4):747-750.

4. Bridges KJ, Bullis CL, Wanchu A, Than KD. Pseudogout of the cervical and thoracic spine mimicking infection after lumbar fusion: case report. J Neurosurg Spine. 2017;27(2):145-149.

5. Mikhael MM, Chioffe MA, Shapiro GS. Calcium pyrophosphate dihydrate crystal deposition disease (pseudogout) of lumbar spine mimicking osteomyelitis-discitis with epidural phlegmon. Am J Orthop. 2013;42(8):E64-E67.

6. Lee J, Cho KT, Kim EJ. Cauda equina syndrome caused by pseudogout involving the lumbar intervertebral disc. J Korean Med Sci. 2012;27(12):1591-1594.

7. Mahmud T, Basu D, Dyson PH. Crystal arthropathy of the lumbar spine: a series of six cases and a review of the literature. J Bone Joint Surg Br. 2005;87(4):513-517. 
8. Andres TL, Trainer TD. Intervertebral chondrocalcinosis: a coincidental finding possibly related to previous surgery. Arch Pathol Lab Med. 1980;104(5):269-271.

9. Dau J, Ho G, Choi H, et al. POS1150 Anatomical locations and correlates of calcium pyrophosphate crystal deposits of the spine pathologic examination of 77 surgical cases [abstract]. Ann Rheum Dis. 2021;80(Suppl 1):854.

10. Gewolb DP, Entezami P, German JW, Narayan A. Rare presentation of intradural calcium pyrophosphate dihydrate crystal deposition. Neuroradiology. 2021;63(10):1735-1737.

11. Chakravorty A, Murambi RT, Cherukuri RKV. Intradural calcium pyrophosphate dihydrate deposition disease: case report. J Neurosurg Spine. 2021;34(5):804-807.

12. Sarubbo S, Garofano F, Maida G, Fainardi E, Granieri E, Cavallo MA. Spontaneous and idiopathic chronic spinal epidural hematoma: two case reports and review of the literature. Eur Spine J. 2009;18(11):1055-1061.

13. Fuster S, Castañeda S, Ferrer E, Wang J, Poblete J. Spontaneous chronic epidural hematoma of the lumbar spine mimicking an extradural spine tumour. Eur Spine J. 2013;22(3 Suppl):S337-S340.

\section{Disclosures}

Dr. Yu reported receiving personal fees from Medtronic and personal fees from Synaptive Medical outside the submitted work. The other authors report no conflict of interest.

\section{Author Contributions}

Conception and design: all authors. Acquisition of data: Jaffee, Elhamdani, Yu. Analysis and interpretation of data: Jaffee, Elhamdani, Yu. Drafting the article: all authors. Critically revising the article: all authors. Reviewed submitted version of manuscript: Jeong, Jaffee, Yu. Approved the final version of the manuscript on behalf of all authors: Jeong. Statistical analysis: Jaffee. Administrative/technical/material support: Jaffee, Yu. Study supervision: Jaffee, Yu.

\section{Correspondence}

Seung Won Jeong: Allegheny General Hospital, Pittsburgh, PA. seung. jeong@ahn.org. 